\title{
SELF-HEATING IN A COUPLED THERMO-ELECTRIC CIRCUIT-DEVICE MODEL*
}

\author{
MARKUS BRUNK ${ }^{\dagger}$ AND ANSGAR JÜNGEL ${ }^{\ddagger}$
}

\begin{abstract}
The self-heating of a coupled thermo-electric circuit-semiconductor system is modeled and numerically simulated. The system consists of semiconductor devices, an electric network with resistors, capacitors, inductors, and voltage sources, and a thermal network. The flow of the charge carriers is described by the energy-transport equations coupled to a heat equation for the lattice temperature. The electric circuit is modeled by the network equations from modified nodal analysis coupled to a thermal network describing the evolution of the temperatures in the lumped and distributed circuit elements. The three subsystems are coupled through thermo-electric, electric circuit-device, and thermal network-device interface conditions. The resulting system of nonlinear partial differential-algebraic equations is discretized in time by the 2-stage backward difference formula and in space by a mixed finite-element method. Numerical simulations of a one-dimensional ballistic diode and a frequency multiplier circuit containing a $p n$-junction diode illustrate the heating of the semiconductor device and circuit resistors.
\end{abstract}

Key words. Energy-transport equations, lattice heating, thermal network, circuit equations, mixed finite-element method, partial differential-algebraic equations.

AMS subject classifications. 65L80, 65M60, 82D37.

1. Introduction. Due to growing package densities, self-heating becomes more and more important in modern integrated circuits and power devices. Thermal effects may strongly influence the device behavior and even reduce its performance. In order to understand the influence of self-heating, power dissipation and temperature evolution have to be taken into account in the electric network models.

In industrially used circuit simulators, complex semiconductor device models are usually substituted by circuits of basic network elements, resulting in simpler so-called compact models, and thermal effects are described heuristically by correction factors or simple heat models. In order to achieve accurate simulations of modern circuits, however, a very large number of circuit elements and a careful adjustment of a large number of parameters is needed. Therefore, it is preferable to model those devices which are critical for the parasitic effects by semiconductor transport equations and to include physical heat flow models.

Beacuse of missing structural informations about the coupled device-circuit equations, first approaches to couple circuits and devices were based on a combination of device and circuit simulators as "black box" solvers [16] or on simple extensions of device simulators by more complex boundary conditions [26]. More recently, electric network models were coupled to semiconductor transport equations, such as driftdiffusion [34] or energy-transport models [11], leading to a coupled system of partial differential-algebraic equations. The work [11] includes temperature models for the

*The authors thank Giuseppe Alì for very fruitful discussions on the thermal network-device coupling and the paper [3]. The authors acknowledge partial support from the German Federal Ministry of Education and Research (BMBF), grant 03JUNAVN. The first author was supported by the ERCIM "Alain Bensoussan" Fellowship Programme. The second author was supported by the Austrian Science Fund (FWF), grant P20214 and WK "Differential Equations", and from the German Science Foundation (DFG), grant JU 359/7. This research is part of the ESF program "Global and geometrical aspects of nonlinear partial differential equations (GLOBAL)".

${ }^{\dagger}$ Department of Mathematical Sciences, Norwegian University of Science and Technology, 7491 Trondheim, Norway; e-mail: markus.brunk@math.ntnu.no.

${ }^{\ddagger}$ Institute for Analysis and Scientific Computing, Vienna University of Technology, Wiedner Hauptstr. 8-10, 1040 Wien, Austria; e-mail: juengel@anum.tuwien.ac.at. 
charge carriers, but the lattice and circuit element temperatures were assumed to be constant.

Nonisothermal device modeling started in the 1970s, employing drift-diffusiontype equations and heat flow models for the lattice temperature $[1]$. In $[17,35]$, a Joule heating term was suggested as source term for the heat flow equation. More sophisticated but still heuristic source term models were presented in $[1,14,33]$. Wachutka employed a thermodynamic approach to extend the drift-diffusion equations to the nonisothermal case [39]. Based on first principles of entropy maximization and partial local equilibrium, Albinus et al. [2] derived nonisothermal carrier transport equations and included also carrier temperatures. Thermal effects in electric circuits were considered in $[8,9]$. Furthermore, an energy-transport model taking into account the heat transfer between the devices and the circuit was proposed in [3].

Models for different lattice and charge carrier temperatures were developed in the 1990s. For instance, the unipolar hydrodynamic semiconductor equations were coupled to a heat equation, with a coupling realized through the energy relaxation term $[18,24]$. In [40], the heat equation and the energy-transport models for the carrier subsystems were coupled.

All the above references are concerned with the modeling of certain subsystems, either neglecting carrier energy models, the coupling to circuits, or lattice heating. In this paper, we propose and simulate for the first time a complete coupled thermo-electric circuit-device model, consisting of (i) energy-transport equations for the charge carriers and the electron temperature in the semiconductor device, together with a heat equation for the lattice temperature, (ii) electric network equations resulting from modified nodal analysis, and (iii) a thermal network model describing the heat evolution in the lumped elements (for instance, resistors, contact nodes, or interconnects) and distributed elements (for instance, electric lines or devices). The three subsystems are coupled by thermo-electric, electric circuit-device, and thermal network-device interfaces. Figure 1.1 presents an overview of the subsystems.

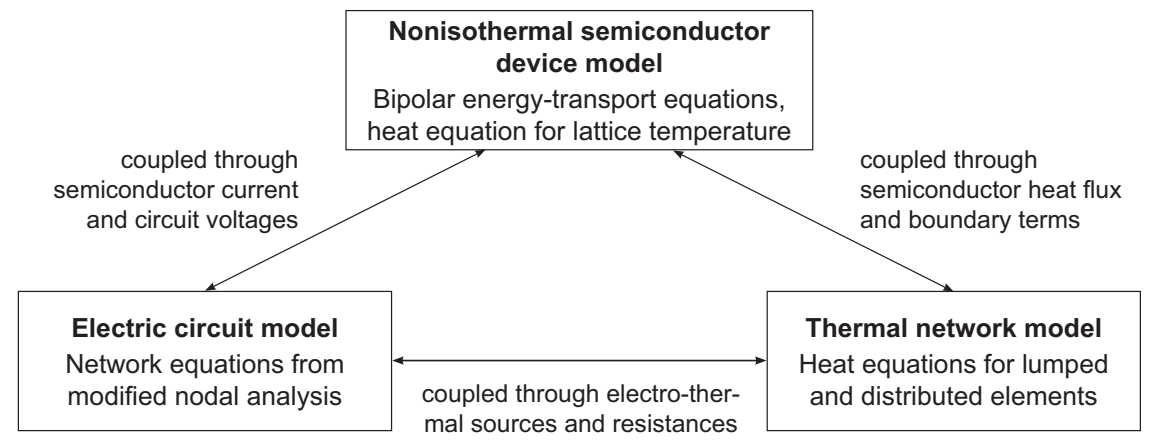

FIG. 1.1. Overview of the coupled thermo-electric circuit-device model.

The equations for the energy-transport model [22], the electric circuit model [38], and the thermal network model [7] are well established. The thermo-electric coupling is described in [3, 7] and the electric circuit-device coupling is detailed in $[11,34]$. In the following, we explain our modeling of the lattice heating and the coupling between the thermal network and semiconductor devices, which seems to be new in this context.

The heat equation for the lattice temperature is derived from thermodynamic principles following the approach of $[2,6]$. We adapt the free energy to the case of 
several temperatures and achieve a heat equation which guarantees energy conservation, i.e., the total energy is assumed to satisfy the balance equation

$$
\partial_{t} u+\operatorname{div} J_{u}=-\gamma
$$

where $J_{u}$ is the corresponding energy flux (density) and $\gamma$ the radiation to the environment. Assuming nondegenerate semiconductors and neglecting the space dependency of the energy bands, the resulting heat equation for the lattice temperature $T_{L}$ is of the type

$$
\rho_{L} c_{L} \partial_{t} T_{L}-\operatorname{div}\left(\kappa_{L} \nabla T_{L}\right)=H
$$

where $\rho_{L}$ is the material density, $c_{L}$ the heat capacity, and $\kappa_{L}$ the heat conductivity of the lattice, according to Fourier's law. The heat source term $H$ contains energy relaxation, recombination heat, and radiation effects (see section 2.2 for details).

The semiconductor device influences the thermal network by the heat fluxes through the contact boundaries, defined by the boundary integral over the normal component of the semiconductor heat flux density $J_{\text {th }}^{S}$. In order to derive $J_{\text {th }}^{S}$ we make a quasi-stationarity assumption. From the stationary energy balance equation without radiation, $\operatorname{div} J_{u}=0$, we infer that

$$
\operatorname{div} J_{\mathrm{th}}^{S}=-\nabla V \cdot\left(J_{n}+J_{p}\right),
$$

where $-\nabla V$ is the electric field and $J_{n}, J_{p}$ are the electron and hole current densities, respectively. This equation indicates that the flux $J_{\mathrm{th}}^{S}$ is responsible for the heat production caused by the dissipated power and can be considered as a heat flux. Detailing the contributions of the energy flux $J_{u}$ yields a formula for $J_{\mathrm{th}}^{S}$, consisting of a heat flux according to the Fourier law, the energy flux of the charge carriers, and the flux of the thermal energy of the carriers (see section 3.5 for details).

The model (with constant hole temperature) is numerically discretized and applied to two examples. In the first example, we present the evolution of the lattice temperature in a one-dimensional ballistic diode. The second example is concerned with transient simulations of the temperatures of the electrons and the lattice in the $p n$-junction diode and the resistor temperatures in a frequency multiplier circuit.

The paper is organized as follows. The following section is concerned with the device modeling including the equation for the lattice heating. In section 3 the lumped and distributed thermal elements are defined and the thermal network model is detailed. The circuit model based on the Kirchhoff laws and current-voltage characteristics of the circuit elements is explained in section 4. The complete coupled model system is summarized and scaled in section 5 . In section 6 we explain the numerical scheme and present the numerical examples. Finally, we conclude in section 7 .

2. Device modeling. In this section we specify the energy-transport model and the heat equation for the lattice temperature, together with the corresponding coupling and the boundary conditions.

2.1. Energy-transport equations. The evolution of the particle and current densities in the semiconductor device is modeled by the bipolar energy-transport equations which can be derived from the semiconductor Boltzmann equation by a moment method [10]. Assuming that the energy band is approximately parabolic, that nondegenerate semiconductor statistics can be used, and that inelastic phonon collisions in the Boltzmann equation can be described by a Fokker-Planck approximation [22], an 
energy-transport model with explicit diffusion coefficients was presented in [15]. We write these equations for the electron density $n$, the hole density $p$, the electron energy density $\frac{3}{2} k_{B} n T_{n}$, and the hole energy density $\frac{3}{2} k_{B} p T_{p}$ with the Boltzmann constant $k_{B}$ and the electron and hole temperatures $T_{n}$ and $T_{p}$, respectively, as follows:

$$
\begin{aligned}
\partial_{t} n-q^{-1} \operatorname{div} J_{n} & =-R(n, p,) \\
\partial_{t} p+q^{-1} \operatorname{div} J_{p} & =-R(n, p), \\
\partial_{t}\left(\frac{3}{2} k_{B} n T_{n}\right)-\operatorname{div} S_{n} & =-\nabla V \cdot J_{n}+W_{n}\left(n, T_{n}\right)-\frac{3}{2} k_{B} T_{n} R(n, p), \\
\partial_{t}\left(\frac{3}{2} k_{B} p T_{p}\right)+\operatorname{div} S_{p} & =-\nabla V \cdot J_{p}+W_{p}\left(p, T_{p}\right)-\frac{3}{2} k_{B} T_{p} R(n, p),
\end{aligned}
$$

where the electric potential $V$ is computed self-consistently from the Poisson equation

$$
\varepsilon_{s} \Delta V=q(n-p-C(x)), \quad x \in \Omega, t>0 .
$$

Here, $\varepsilon_{s}$ denotes the semiconductor permittivity, $q$ the elementary charge, $C(x)$ the doping concentration characterizing the device under consideration, and $\Omega \subset \mathbb{R}^{d}$ $(d \geq 1)$ the domain occupied by the semiconductor.

The electron and hole current densities $J_{n}$ and $J_{p}$, respectively, and the corresponding energy current densities $S_{n}$ and $S_{p}$ are given by

$$
\begin{aligned}
J_{n} & =q\left(\nabla\left(\mu_{n}\left(T_{L}\right) \frac{k_{B} T_{L}}{q} n\right)-\mu_{n}\left(T_{L}\right) T_{L} \frac{n}{T_{n}} \nabla V\right), \\
J_{p} & =-q\left(\nabla\left(\mu_{p}\left(T_{L}\right) \frac{k_{B} T_{L}}{q} p\right)+\mu_{p}\left(T_{L}\right) T_{L} \frac{p}{T_{p}} \nabla V\right), \\
S_{n} & =\nabla\left(\frac{3}{2} \mu_{n}\left(T_{L}\right) T_{n} \frac{k_{B}^{2} T_{L}}{q} n\right)-\frac{3}{2} \mu_{n}\left(T_{L}\right) n k_{B} T_{L} \nabla V, \\
S_{p} & =-\nabla\left(\frac{3}{2} \mu_{p}\left(T_{L}\right) T_{p} \frac{k_{B}^{2} T_{L}}{q} p\right)-\frac{3}{2} \mu_{p}\left(T_{L}\right) p k_{B} T_{L} \nabla V,
\end{aligned}
$$

where $T_{L}$ denotes the (nonconstant) lattice temperature and $\mu_{n}$ and $\mu_{p}$ are the electron and hole mobilities, respectively, which are assumed to depend on the lattice temperature according to the power law

$$
\mu_{\nu}\left(T_{L}\right)=\mu_{\nu, 0}\left(\frac{T_{0}}{T_{L}}\right)^{\alpha_{\nu}}, \quad \nu=n, p
$$

where $T_{0}=300 \mathrm{~K}$. The values $\mu_{\nu, 0}$ and $\alpha_{\nu}(\nu=n, p)$ are typically determined from measurements; see, for instance, [33, Table 4.1-1]. Alternatively, the transport of the holes may be described by the drift-diffusion model as is often done (and as we do in the numerical simulations). In this case, the hole temperature is constant, and the transport equations read as

$$
\partial_{t} p+q^{-1} \operatorname{div} J_{p}=-R(n, p), \quad J_{p}=-q\left(\nabla\left(\mu_{p}\left(T_{L}\right) \frac{k_{B} T_{L}}{q} p\right)+\mu_{p}\left(T_{L}\right) p \nabla V\right) .
$$

Recombination and generation of charge carriers are modeled by Shockley-ReadHall and Auger terms,

$$
R(n, p)=\frac{n p-n_{i}^{2}}{\tau_{p}\left(n+n_{i}\right)+\tau_{n}\left(p+n_{i}\right)}+\left(C_{n} n+C_{p} p\right)\left(n p-n_{i}^{2}\right),
$$


where $n_{i}$ is the intrinsic density, $\tau_{n}$ and $\tau_{p}$ are the material-dependent electron and hole lifetimes, respectively, and $C_{n}$ and $C_{p}$ are the Auger parameters. Auger processes are important if the carrier densities are high. In optoelectronic devices further generation processes occur, see, e.g., [12]. The relaxation to the lattice temperature is described by the expressions

$$
W_{n}\left(n, T_{n}\right)=\frac{3}{2} \frac{n k_{B}\left(T_{L}-T_{n}\right)}{\tau_{n, 0}}, \quad W_{p}\left(p, T_{p}\right)=\frac{3}{2} \frac{p k_{B}\left(T_{L}-T_{p}\right)}{\tau_{p, 0}},
$$

where $\tau_{n, 0}$ and $\tau_{p, 0}$ are the energy relaxation times.

For constant lattice temperature, the above energy-transport equations correspond to the model suggested in [13] and referred to as the Chen model in [15]. Generally, the electron current density can be written as

$$
J_{n}=q \mu_{n}\left(T_{L}\right) \frac{k_{B} T_{L}}{q} \nabla n+q D_{n}\left(T_{L}\right) n \nabla T_{L}-q \mu_{n}\left(T_{L}\right) T_{L} \frac{n}{T_{n}} \nabla V,
$$

where $D_{n}\left(T_{L}\right)$ is the partial derivative of $\mu_{n}\left(T_{L}\right) k_{B} T_{L} / q$ with respect to $T_{L}$. Thus, besides of the electric field and the density gradient, there is an additional driving force due to the lattice temperature gradient. We remark that there exist other energy-transport models with a particle temperature gradient [15]. Whether such a term occurs or not depends on the energy-dependency of the scattering rate of the elastic collision operator. In the Chen model, the dependency is such that the particle temperature gradient vanishes. We refer to [19] for a discussion and to [15] for a numerical comparison of various energy-transport models.

2.2. Lattice heating. We derive an evolution equation for the lattice temperature $T_{L}$ by thermodynamic principles, similar as in [6]. The three subsystems defined by the electron, hole, and lattice temperatures are thermally coupled by mutual energy and heat flux exchange. We assume that the total energy $u$ satisfies the balance equation $\partial_{t} u+\operatorname{div} J_{u}=-\gamma$, where $J_{u}$ is the corresponding energy flux density and $\gamma$ the radiation. Analogous to [6], we define the free energy of the system as

$$
\begin{aligned}
f= & \frac{\varepsilon_{s}}{2}|\nabla V|^{2}+\rho_{L} c_{L} T_{L}\left(1-\log T_{L}\right)+n\left(k_{B} T_{n}\left(\log \frac{n}{N_{c}}-1\right)+E_{c}\right) \\
& +p\left(k_{B} T_{p}\left(\log \frac{p}{N_{v}}-1\right)-E_{v}\right),
\end{aligned}
$$

where $\rho_{L}$ is the material density of the lattice, $c_{L}$ the lattice heat capacity, and $E_{c}$ and $E_{v}$ denote the conduction and valence band-edge energies, respectively. The effective densities of states are given by

$$
N_{c}=2\left(\frac{m_{e}^{*} k_{B} T_{n}}{2 \pi \hbar^{2}}\right)^{3 / 2}, \quad N_{v}=2\left(\frac{m_{h}^{*} k_{B} T_{p}}{2 \pi \hbar^{2}}\right)^{3 / 2}
$$

where $m_{e}^{*}$ and $m_{h}^{*}$ are the effective electron and hole mass, respectively, and $\hbar=h / 2 \pi$ is the reduced Planck constant. The first term in (2.2) is the electric energy, the second term the thermodynamic energy of the lattice subsystem, and the last two terms are the thermodynamic energies of the electron and hole subsystems, respectively.

By thermodynamics, the total internal energy $u$ of the system can be written as

$$
\begin{aligned}
u & =f-T_{n} \frac{\partial f}{\partial T_{n}}-T_{p} \frac{\partial f}{\partial T_{p}}-T_{L} \frac{\partial f}{\partial T_{L}} \\
& =\frac{\varepsilon_{s}}{2}|\nabla V|^{2}+\rho_{L} c_{L} T_{L}+n\left(E_{c}-T_{L} E_{c}^{\prime}\right)+\frac{3}{2} k_{B} n T_{n}+p\left(T_{L} E_{v}^{\prime}-E_{v}\right)+\frac{3}{2} k_{B} p T_{p},
\end{aligned}
$$


where the prime in $E_{c}^{\prime}$ and $E_{v}^{\prime}$ signifies the derivative with respect to $T_{L}$. Thus, the total internal energy $u$ is the sum of the electric energy, $\varepsilon_{s}|\nabla V|^{2} / 2$, the energy of the crystal lattice, $\rho_{L} c_{L} T_{L}$, the thermal energies of the electrons and holes, $\frac{3}{2} n k_{B} T_{n}$ and $\frac{3}{2} p k_{B} T_{p}$, respectively, the energy of the electrons in the conduction band, $n\left(E_{c}-T_{L} E_{c}^{\prime}\right)$, and the energy of the holes in the valence band, $p\left(T_{L} E_{v}^{\prime}-E_{v}\right)$. If all temperatures coincide, $T_{L}=T_{n}=T_{p}$, the expressions for the free and internal energies are the same as in [6] for the drift-diffusion case.

Additionally, we introduce the energy fluxes corresponding to the energy densities:

$$
\begin{aligned}
J_{u}^{E} & =-\varepsilon_{s} V \nabla \partial_{t} V, & J_{u}^{L} & =-\kappa_{L} \nabla T_{L}, \\
S_{u}^{n} & =-S_{n}, & S_{u}^{p} & =S_{p}, \\
J_{u}^{n} & =-q^{-1}\left(E_{c}-T_{L} E_{c}^{\prime}\right) J_{n}+V J_{n}, & J_{u}^{p} & =-q^{-1}\left(E_{v}-T_{L} E_{v}^{\prime}\right) J_{p}+V J_{p},
\end{aligned}
$$

where $\kappa_{L}$ is the heat conductivity of the crystal lattice. The equation for the heat flux $J_{u}^{L}$ expresses the Fourier law. The term $-\varepsilon_{s} \nabla \partial_{t} V$ is the so-called displacement current which has to be taken into account in transient simulations to guarantee charge conservation. The sum of $J_{u}^{E}$ and the second terms in $J_{u}^{n}$ and $J_{u}^{p}$ is the expression for the current of the dissipated power, given as the product of total current and electric potential. The sum of all energy flux densities is the total energy flux density, $J_{u}=J_{u}^{E}+J_{u}^{L}+S_{u}^{n}+S_{u}^{p}+J_{u}^{n}+J_{u}^{p}$.

Inserting the above expressions for the total internal energy $u$ and the total energy flux $J_{u}$ into the energy balance equation and employing the Poisson equation for $\partial_{t} V$,

$$
\varepsilon_{s} \Delta \partial_{t} V=q\left(\partial_{t} n-\partial_{t} p\right)=\operatorname{div}\left(J_{n}+J_{p}\right),
$$

we arrive after a straightforward computation to

$$
0=\partial_{t} u+\operatorname{div} J_{u}+\gamma=\partial_{t} T_{L}\left(\rho_{L} c_{L}-n E_{c}^{\prime}+p E_{v}^{\prime}\right)-\operatorname{div}\left(\kappa_{L} \nabla T\right)-H,
$$

where the heat source term $H$ is given by

$$
\begin{aligned}
H= & -\left(W_{n}+W_{p}\right)+R\left(E_{g}-T_{L} E_{g}^{\prime}+\frac{3}{2} k_{B}\left(T_{n}+T_{p}\right)\right) \\
& +q^{-1} J_{n} \cdot \nabla\left(E_{c}-T_{L} E_{c}^{\prime}\right)+q^{-1} J_{p} \cdot \nabla\left(E_{v}-T_{L} E_{v}^{\prime}\right)-\gamma,
\end{aligned}
$$

and $E_{g}=E_{c}-E_{v}$ is the energy gap.

Now, we impose some simplifying assumptions. For nondegenerate homostructure devices, we may neglect the space dependency of the energy bands. Furthermore, we neglect the dependency of the energy bands on the lattice temperature since this dependency is rather small [33]. Finally, we assume that the radiation is proportional to the deviation of the lattice temperature from the environmental temperature $T_{\text {env }}$. Thus, we obtain the evolution equation for the lattice temperature,

$$
\rho_{L} c_{L} \partial_{t} T_{L}-\operatorname{div}\left(\kappa_{L} \nabla T_{L}\right)=H,
$$

where

$$
H=-\left(W_{n}+W_{p}\right)+R\left(E_{g}+\frac{3}{2} k_{B}\left(T_{n}+T_{p}\right)\right)-S_{L}\left(T_{L}-T_{\text {env }}\right),
$$

and $S_{L}=S_{L}(x)$ denotes the transmission function of the device. For the numerical simulations, we will assume that this function is constant. The first term in $H$ represents the energy relaxation heat, the second term is the recombination heat, and 
the last term signifies the radiation. We remark that this expression for the heat source differs from those in [6] and [39] since we take into account the evolution of the thermal energies of the particles via the energy-transport equations. In [6], only the drift-diffusion equations were considered. The simplified source term (2.4) corresponds essentially to formula (41) of [40]. The source term (2.3) allows for space-dependent energy bands and generalizes the existing approaches.

2.3. Initial and boundary conditions. The boundary $\partial \Omega$ of the semiconductor domain is assumed to consist of two parts, the union of Ohmic contacts $\Gamma_{C}$ and the union of insulating boundary segments $\Gamma_{I}$, where $\partial \Omega=\Gamma_{C} \cup \Gamma_{I}$ and $\Gamma_{C} \cap \Gamma_{I}=\emptyset$. We prescribe initial conditions for the particle densities and temperatures:

$$
n(\cdot, 0)=n_{I}, \quad p(\cdot, 0)=p_{I}, \quad T_{j}(\cdot, 0)=T_{j, I} \quad \text { in } \Omega, t>0, j=n, p, L .
$$

On the insulating parts of the boundary, we suppose that the normal components of the current and energy fluxes, the lattice temperature flux, and the electric field vanish,

$$
J_{j} \cdot \nu=S_{j} \cdot \nu=\nabla T_{L} \cdot \nu=\nabla V \cdot \nu=0 \quad \text { on } \Gamma_{I}, t>0, j=n, p,
$$

where $\nu$ is the exterior normal unit vector on $\partial \Omega$. Instead of requiring that the normal components of the energy fluxes vanish, we can equivalently assume that the temperature fluxes vanish,

$$
\nabla T_{n} \cdot \nu=\nabla T_{p} \cdot \nu=0 \quad \text { on } \Gamma_{I}, t>0 .
$$

The semiconductor contacts connecting the device with the electric network are supposed to be Ohmic semiconductor-metal contacts. Then the electric potential equals on $\Gamma_{C}$ the sum of the applied potential $V_{\text {app }}$ and the built-in potential $V_{\mathrm{bi}}$ (see, e.g., [30]):

$$
V=V_{\mathrm{app}}+V_{\mathrm{bi}} \quad \text { on } \Gamma_{C}, t>0, \quad \text { where } V_{\mathrm{bi}}=\operatorname{arcsinh}\left(\frac{C(x)}{2 n_{i}}\right) .
$$

We assume that there is no exchange of the particle temperatures $T_{n}$ and $T_{p}$ across the Ohmic contacts such that the use of homogeneous Neumann conditions for the temperatures seems to be reasonable. In fact, Dirichlet conditions for $T_{n}$ and $T_{p}$ may lead to artificial boundary layers [11]. Moreover, in [5] it was argued that the use of homogeneous Neumann conditions for $T_{n}$ and $T_{p}$ can be justified in highly doped regions close to the contacts. For the lattice temperature, we employ Robin boundary conditions in order to model the temperature exchange between the semiconductor device and the connected thermal and circuit elements. Therefore, we have

$$
\nabla T_{n} \cdot \nu=0, \quad \nabla T_{p} \cdot \nu=0, \quad-\kappa_{L} \nabla T_{L} \cdot \nu=R_{\mathrm{th}}^{-1}\left(T_{L}-T_{a}\right) \quad \text { on } \Gamma_{C}, t>0,
$$

where $R_{\mathrm{th}}$ denotes the thermal resistance of the contact and $T_{a}$ is the temperature of the connected element (defined below in (3.5)).

Concerning the boundary conditions for the particle densities at the contacts, Yamnahakki [41] showed for the drift-diffusion model that the densities are given by

$$
n+\left(\theta_{n} \mu_{n}\right)^{-1} J_{n} \cdot \nu=n_{a}, \quad p-\left(\theta_{p} \mu_{p}\right)^{-1} J_{p} \cdot \nu=p_{a} \quad \text { on } \Gamma_{C}, t>0,
$$

with some parameters $\theta_{n}$ and $\theta_{p}$, the temperature-dependent mobilities $\mu_{n}, \mu_{p}$ are defined in (2.1), and the densities $n_{a}$ and $p_{a}$ are determined under the assumptions 
of charge neutrality, $n_{a}-p_{a}-C(x)=0$, and thermal equilibrium at the contacts, $n_{a} p_{a}=n_{i}^{2}$, leading to

$$
n_{a}=\frac{1}{2}\left(C(x)+\sqrt{C(x)^{2}+4 n_{i}^{2}}\right), \quad p_{a}=\frac{1}{2}\left(-C(x)+\sqrt{C(x)^{2}+4 n_{i}^{2}}\right) .
$$

The boundary conditions (2.6) were derived from a kinetic boundary condition for the semiconductor Boltzmann equation up to second order in the Knudsen number [41]. They give reasonable results in numerical simulations even when the bipolar energy-transport equations were employed [11], and we use them also in our model.

3. Thermal network modeling. In this section we describe the thermal network based on the model of [7,9]. The thermal network describes how the heat is evolving in the semiconductor-circuit topology. To simplify the presentation, we consider only one semiconductor device in the network. The extension to several semiconductor devices is straightforward.

3.1. Definition of distributed and lumped elements. As a compromise between physical accuracy and fast numerical simulations, all thermally relevant elements in the circuit - except the semiconductor devices - are modeled by zero- or one-dimensional structures. More precisely, we consider the following elements in the thermal network.

Lumped thermal elements are zero-dimensionally modeled elements to which a spatially constant temperature value $\widehat{T}^{\ell}(t)$ is associated. They can be used if, for instance, the temperature is approximately constant in a circuit element due to small extension or large thermal conductivity. Some circuit elements may be modeled as distributed thermal lines. The associated temperature $T^{d}(x, t)$ depends on the onedimensional space variable $x \in\left[0, L_{\mathrm{th}}\right]$ and on time $t>0$. The contacts of such an element are at $x=0$ and $x=L_{\mathrm{th}}$. Finally, we consider distributed semiconductor devices with the lattice temperature $T_{L}(x, t)$ as described in the previous section.

The distributed elements are coupled by the requirement that the temperature and its heat flux across the boundary of the elements are continuous. Accordingly, adjacent lumped elements are considered as zero-dimensional units with one temperature value $\widehat{T}$. Then, we assign the temperature at the interfaces of connected distributed elements to an artificial zero-dimensional unit without thermal mass. In this way, we end up with a thermal network only containing lumped-distributed interfaces. The nodes of this network are represented by the zero-dimensional units and the branches by the distributed elements. We remark that the resulting network may not be connected.

The thermal network with $m_{n}$ thermal nodes, $m_{d}$ thermal lines (branches), and one semiconductor device with $n_{S}$ terminals can be characterized by the thermal incidence matrix $\mathcal{A}_{d}^{\text {th }}=\left(a_{i j}^{\text {th }}\right) \in \mathbb{R}^{m_{n} \times 2 m_{d}}$ and the thermal semiconductor incidence $\operatorname{matrix} \mathcal{A}_{S}^{\text {th }}=\left(a_{S, i j}^{\text {th }}\right) \in \mathbb{R}^{m_{n} \times n_{S}}$ defined by

$$
\begin{aligned}
a_{i j}^{\mathrm{th}} & = \begin{cases}1 & \text { if the contact at } x=0 \text { of branch } j \text { is connected to node } i, \\
1 & \text { if the contact at } x=L_{\mathrm{th}} \text { of branch } j-m_{d} \text { is connected to node } i, \\
0 & \text { else, }\end{cases} \\
a_{S, i j}^{\mathrm{th}} & = \begin{cases}1 & \text { if the terminal } j \text { is connected to thermal node } i, \\
0 & \text { else. }\end{cases}
\end{aligned}
$$

These definitions of the thermal incidence matrices without using any description 
from the electric network allow for a flexible modeling of thermally distributed electric network nodes.

3.2. Temperature in lumped elements and thermal nodes. The lumped elements are related to the thermal nodes by means of the matrix $\mathcal{M}=\left(m_{i j}\right) \in$ $\mathbb{R}^{m_{n} \times m_{\ell}}$, where $m_{\ell}$ is the number of lumped thermal elements, in the following way:

$$
m_{i j}= \begin{cases}1 & \text { if the lumped element } j \text { belongs to the thermal node } i, \\ 0 & \text { else. }\end{cases}
$$

The thermal mass $\widehat{M}$ of a lumped thermal element is the product of the heat capacity $\widehat{c}^{\ell}$, the material density $\widehat{\rho}^{\ell}$, and the physical volume $\widehat{V}^{\ell}, \widehat{M}=\widehat{c}^{\ell} \widehat{\rho}^{\ell} \widehat{V}^{\ell}$. Let $m_{\ell}$ (artificial) lumped thermal elements with thermal masses $\widehat{M}_{1}, \ldots, \widehat{M}_{m_{\ell}}$, transmission functions $\widehat{S}_{1}, \ldots, \widehat{S}_{m_{\ell}}$, and thermo-electric sources $\widehat{P}_{1}, \ldots, \widehat{P}_{m_{\ell}}$ (defined in (4.7)) be given. With the matrix $\mathcal{M}$, we define the diagonal matrix $\widehat{M}$, containing the thermal masses, and the transmission function $\widehat{\boldsymbol{S}}$ for the thermal nodes (zero-dimensional units) by

$$
\widehat{\boldsymbol{M}}=\mathcal{M} \operatorname{diag}\left(\widehat{M}_{1}, \ldots, \widehat{M}_{m_{\ell}}\right) \mathcal{M}^{\top}, \quad \widehat{\boldsymbol{S}}=\mathcal{M} \operatorname{diag}\left(\widehat{S}_{1}, \ldots, \widehat{S}_{m_{\ell}}\right) \mathcal{M}^{\top} .
$$

The electro-thermal sources for the thermal nodes and the heat flux from the distributed lines and devices into the thermal nodes are collected in the vectors

$$
\widehat{\boldsymbol{P}}=\mathcal{M}\left(\widehat{P}_{1}, \ldots, \widehat{P}_{m_{\ell}}\right)^{\top}, \quad \widehat{\boldsymbol{F}}^{d}=\left(\widehat{F}_{1}^{d}, \ldots, \widehat{F}_{m_{n}}^{d}\right)^{\top}, \quad \text { and } \quad \widehat{\boldsymbol{F}}^{S},
$$

defined below in (4.7), (3.4), and (3.6)-(3.7), respectively. Furthermore, the temperature values of the thermal nodes are collected in the vector $\widehat{T}$ and we assume that it evolves according to the heat equation

$$
\widehat{\boldsymbol{M}} \frac{d \widehat{\boldsymbol{T}}}{d t}=\widehat{\boldsymbol{F}}^{d}+\widehat{\boldsymbol{F}}^{S}-\widehat{\boldsymbol{S}}\left(\widehat{\boldsymbol{T}}-T_{\mathrm{env}} \boldsymbol{I}\right)+\widehat{\boldsymbol{P}}
$$

where $\widehat{\boldsymbol{S}}$ is the transmission function and $\boldsymbol{I}$ the identity matrix. The temperature values in the lumped elements $\widehat{\boldsymbol{T}}^{\ell}$ can be recovered from the temperature values in the thermal nodes $\widehat{\boldsymbol{T}}$ by the formula $\widehat{\boldsymbol{T}}=\mathcal{M} \widehat{\boldsymbol{T}}^{\ell}$.

3.3. Temperature in distributed elements and thermal branches. The vector $\boldsymbol{T}^{d}=\left(T_{1}^{d}, \ldots, T_{m_{d}}^{d}\right)$ of all temperature values of the thermal lines satisfies the heat equation

$$
M_{j} \partial_{t} T_{j}^{d}=\partial_{x}\left(\kappa_{j} \partial_{x} T_{j}^{d}\right)-S_{j}\left(T_{j}^{d}-T_{\mathrm{env}}\right)+P_{j}, \quad(x, t) \in\left(0, L_{j}\right) \times[0, \infty),
$$

where $j=1, \ldots, m_{d}$ and $L_{j}$ is the length of the $j$-th thermal line. Here, $M_{j}=c_{j}^{d} \rho_{j}^{d} A_{j}^{d}$ denotes the thermal mass, given as the product of the heat capacity $c_{j}^{d}$, the material density $\rho_{j}^{d}$, and the area of the cross section $A_{j}^{d}$ of the considered element. Furthermore, $\kappa_{j}$ is the thermal conductivity, $S_{j}$ the transmission function which describes the thermal radiation to the environment and which is supposed to be proportional to the physical surface of the modeled device, and $P_{j}$ denotes the electro-thermal source term, defined below in (4.7). Equation (3.2) is complemented by the initial and boundary conditions

$$
\begin{aligned}
T_{j}^{d}(x, 0) & =T_{I, j}^{d}(x), \quad x \in\left[0, L_{j}\right], j=1, \ldots, m_{d}, \\
T_{j}^{d}(0, t) & =T_{0, j}^{d}(t), \quad T_{j}^{d}\left(L_{j}, t\right)=T_{1, j}^{d}(t), \quad t \in[0, \infty) .
\end{aligned}
$$


We collect all boundary values in the vectors $\boldsymbol{T}_{0}^{d}(t)=\left(T_{0,1}^{d}, \ldots, T_{0, m_{d}}^{d}\right)(t)$ and $\boldsymbol{T}_{1}^{d}(t)=$ $\left(T_{1,1}^{d}, \ldots, T_{1, m_{d}}^{d}\right)(t)$ and we set $\boldsymbol{T}^{d}=\left(T_{1}^{d}, \ldots, T_{m_{d}}^{d}\right)$.

3.4. Coupling between thermal nodes and branches. The heat equations for the lumped elements and the distributed lines are coupled in the following way. First, we assume that the temperature of the thermal lines at the boundary contacts coincides with the temperature $\widehat{\boldsymbol{T}}$ of the adjacent thermal nodes. This condition can be expressed by means of the thermal incidence matrix introduced in section 3.1:

$$
\left(\boldsymbol{T}_{0}^{d}, \boldsymbol{T}_{1}^{d}\right)^{\top}=\left(\mathcal{A}_{d}^{\mathrm{th}}\right)^{\top} \widehat{\boldsymbol{T}}
$$

Second, we suppose that the thermal flux is given by the Fourier law,

$$
\widehat{\boldsymbol{F}}^{d}=\mathcal{A}_{d}^{\mathrm{th}}\left(\begin{array}{c}
\Lambda(0) \partial_{x} \boldsymbol{T}^{d}(0, t) \\
-\Lambda\left(L_{\mathrm{th}}\right) \partial_{x} \boldsymbol{T}^{d}\left(L_{\mathrm{th}}, t\right)
\end{array}\right),
$$

where $\Lambda(x)=\operatorname{diag}\left(A_{1}^{d} \kappa_{1}(x), \ldots, A_{m_{d}}^{d} \kappa_{m_{d}}(x)\right), A_{j}^{d}$ denotes the cross section and $\kappa_{j}$ the thermal conductivity of the $j$-th thermal line. Then the vector $\widehat{\boldsymbol{F}}^{d}=\left(\widehat{F}_{1}^{d}, \ldots, \widehat{F}_{m_{n}}^{d}\right)$ enters the lumped heat equation (3.1).

3.5. Coupling between thermal network and semiconductor device. The semiconductor device is included into the thermal network by means of the thermal semiconductor matrix introduced in section 3.1. In analogy to the thermal line modeling, the device equations are coupled to the thermal nodes using the boundary conditions for the temperature and the heat flux. Thus, the last boundary condition in $(2.5)$ changes to

$$
-\kappa_{L} \nabla T_{L} \cdot \nu=R_{\mathrm{th}}^{-1}\left(T_{L}-T_{a, k}\right) \quad \text { on } \Gamma_{k}, k=1, \ldots, n_{S},
$$

where $\Gamma_{k} \subset \Gamma_{C}$ is the $k$-th contact of the device, and the temperature of the connected elements is defined by

$$
\boldsymbol{T}_{a}=\left(T_{a, 1}, \ldots, T_{a, n_{S}}\right)=\left(\mathcal{A}_{S}^{\mathrm{th}}\right)^{\top} \widehat{\boldsymbol{T}}
$$

This describes the influence of the thermal network on the lattice temperature. The influence of the semiconductor heat flux on the thermal network could be described in a first approximation by the Fourier law, with a coupling analogous to the coupling between thermal nodes and branches (see (3.4)). However, the flow of hot electrons, modeled by the energy-transport equations, contributes to the heat flux and has to be taken into account (see [3]). We assume that the semiconductor heat flux at terminal $k$ is given by the boundary integral over the normal component of the thermal flux density:

$$
F_{k}^{S}=\int_{\Gamma_{k}} J_{\mathrm{th}}^{S} \cdot \nu d \sigma, \quad k=1, \ldots, n_{S},
$$

such that the heat flux vector $\widehat{\boldsymbol{F}}^{S}$, used in (3.1), becomes

$$
\widehat{\boldsymbol{F}}^{S}(t)=\mathcal{A}_{S}^{\mathrm{th}}\left(F_{1}^{S}(t), \ldots, F_{n_{S}}^{S}(t)\right), \quad t>0 .
$$

In order to specify the thermal flux density $J_{\mathrm{th}}^{S}$, we make a quasi-stationarity assumption. Without radiation, the stationary balance equation for the total energy reads as (see section 2.2 )

$$
0=\operatorname{div} J_{u}=\operatorname{div} J_{u}^{E}+\operatorname{div}\left(J_{u}^{L}+S_{u}^{n}+S_{u}^{p}+J_{u}^{n}+J_{u}^{p}\right) .
$$


Now, the balance equation for the electric energy can be written as

$$
\partial_{t} u_{E}+\operatorname{div} J_{u}^{E}=-\varepsilon_{s} V \Delta \partial_{t} V=-V \partial_{t}(n-p)=-V \operatorname{div}\left(J_{n}+J_{p}\right) .
$$

In the quasi-stationary approximation, we insert $\operatorname{div} J_{u}^{E}=-V \operatorname{div}\left(J_{n}+J_{p}\right)$ in the above conservation law for $J_{u}$ :

$$
\begin{aligned}
0= & -V \operatorname{div}\left(J_{n}+J_{p}\right)+\operatorname{div}\left(J_{u}^{L}+S_{u}^{n}+S_{u}^{p}+J_{u}^{n}+J_{u}^{p}\right) \\
= & -V \operatorname{div}\left(J_{n}+J_{p}\right) \\
& +\operatorname{div}\left(-\kappa_{L} \nabla T_{L}-S_{n}+S_{p}-q^{-1}\left(E_{c} J_{n}+E_{v} J_{p}\right)+V\left(J_{n}+J_{p}\right)\right) \\
= & \operatorname{div} J_{\mathrm{th}}^{S}+\nabla V \cdot\left(J_{n}+J_{p}\right),
\end{aligned}
$$

where we have assumed as in section 2.2 that the energy bands do not depend on the lattice temperature and we have defined the flux

$$
J_{\mathrm{th}}^{S}=-\kappa_{L} \nabla T_{L}-q^{-1}\left(E_{c} J_{n}+E_{v} J_{p}\right)-S_{n}+S_{p} .
$$

Equation (3.8) indicates that the flux $J_{\text {th }}^{S}$ is responsible for the heat production caused by the dissipated power and can be therefore considered as a heat flux. The first term in the definition of $J_{\text {th }}^{S}$ describes the heat flux according to the Fourier law, as it occurs in the thermal lines; the second term determines the energy flux of the charge carriers with energy $E_{c}$ or $E_{v}$ per carrier; and the last term describes the flux of the thermal energy of the particles. We notice that in [3], slightly different energytransport equations compared to the model in section 2.1 were considered. The heat flux of [3] coincides with the electron energy flux $S_{n}$ of our model. In this sense, our model is an extension of the model in [3].

The thermal network equations (3.1), (3.2), and (3.3) have to be complemented by initial conditions for the temperature variables,

$$
\widehat{\boldsymbol{T}}(0)=\widehat{\boldsymbol{T}}_{I}, \quad T_{j}^{d}(\cdot, 0)=T_{I, j}^{d} \quad \text { for } x \in\left(0, L_{j}\right), j=1, \ldots, m_{d} .
$$

We remark that the system (3.1)-(3.4) is of partial differential-algebraic type if the corresponding thermal network contains artificial lumped elements. In this case the initial values have to be consistent (in the sense of the theory of differential-algebraic equations).

4. Circuit modeling. The circuits considered in this paper contain only (ideal) resistors, capacitors, inductors, and voltage sources. In addition, we use ideal current sources. Again, the circuit is assumed to contain only one semiconductor device.

4.1. Modified nodal analysis. A well-established mathematical description of RCL circuits (just containing resistors, capacitors, and inductors) is the modified nodal analysis. The basic tools are the Kirchhoff laws and the current-voltage characteristics of the basic elements. In order to accomplish the modified nodal analysis, the circuit is replaced by a directed graph with branches and nodes. Branch currents, branch voltages, and node potentials (without the mass node) are introduced as (time-dependent) variables. Then the circuit can be characterized by the electrical incidence matrix $\mathcal{A}=\left(a_{k \ell}\right)$ describing the node-to-branch relations and defined by

$$
a_{k \ell}=\left\{\begin{aligned}
1 & \text { if the branch } \ell \text { leaves the node } k \\
-1 & \text { if the branch } \ell \text { enters the node } k \\
0 & \text { else. }
\end{aligned}\right.
$$


(see [38] for details on circuit topologies). The semiconductor device is included into the network model by means of the electrical semiconductor incidence matrix $\mathcal{A}_{S}=\left(a_{k \ell}^{S}\right)$ defined by

$$
a_{k \ell}^{S}=\left\{\begin{aligned}
1 & \text { if the current } j_{\ell} \text { enters the circuit node } k \\
-1 & \text { if the reference terminal is connected to the node } k \\
0 & \text { else. }
\end{aligned}\right.
$$

The semiconductor current density consists of three parts, the electron current density $J_{n}$, the hole current density $J_{p}$, and the displacement current density $J_{d}=$ $-\varepsilon_{s} \nabla \partial_{t} V$. The current leaving the semiconductor device at terminal $k$ is defined by

$$
j_{k}=\int_{\Gamma_{k}} J_{\mathrm{tot}} \cdot \nu d s, \quad \text { where } J_{\mathrm{tot}}=J_{n}+J_{p}+J_{d}, k=1, \ldots, n_{S} .
$$

Clearly, due to charge conservation, the current through one terminal can be computed by the negative sum of the currents through all other terminals. Therefore,we choose one terminal (usually the bulk terminal) as the reference terminal. We denote by $j_{S}$ the vector of all terminal currents except the current corresponding to the reference terminal.

The first tool for the circuit modeling are the current-voltage characteristics for the basic elements,

$$
i_{R}=g_{R}\left(v_{R}\right), \quad i_{C}=\frac{d q_{C}}{d t}\left(v_{C}\right), \quad v_{L}=\frac{d \phi_{L}}{d t}\left(i_{L}\right),
$$

where $g_{R}$ denotes the conductivity of the resistor, $q_{C}$ the charge of the capacitor, and $\phi_{L}$ the flux of the inductor. Moreover, $i_{\alpha}$ and $v_{\alpha}$ with $\alpha=R, C, L$, are the branch current vectors and branch voltage vectors for, respectively, all resistors, capacitors, and inductors. The network branches are numbered in such a way that the incidence matrix forms a block matrix with blocks describing the different types of network elements, i.e., $\mathcal{A}$ consists of the block matrices $\mathcal{A}_{R}, \mathcal{A}_{C}, \mathcal{A}_{L}, \mathcal{A}_{i}$, and $\mathcal{A}_{v}$, where the index indicates the resistive, capacitive, inductive, current source, and voltage source branches, respectively.

The second tool are the Kirchhoff laws. The current law expresses that the sum of all branch currents entering a node is equal to zero, $\mathcal{A} i=0$, and the voltage law means that the sum of all branch voltages in a loop vanishes, $v=\mathcal{A}^{\top} e$, where $i, v$, and $e$ are the vectors of branch currents, branch voltages, and node potentials, respectively. Denoting by $i_{s}=i_{s}(t)$ and $v_{s}=v_{s}(t)$ the given input functions for the current and voltage sources, respectively, and replacing the branch currents in the Kirchhoff current law by the current-voltage characteristics and the branch voltages by node potentials using the Kirchhoff voltage law, we obtain the system in the charge-oriented modified nodal analysis approach [38],

$$
\begin{aligned}
\mathcal{A}_{C} \frac{d q_{C}}{d t}\left(\mathcal{A}_{C}^{\top} e\right)+\mathcal{A}_{R} g_{R}\left(\mathcal{A}_{R}^{\top} e\right)+\mathcal{A}_{L} i_{L}+\mathcal{A}_{v} i_{v}+\mathcal{A}_{S} j_{S} & =-\mathcal{A}_{i} i_{s}, \\
\frac{d \phi_{L}}{d t}\left(i_{L}\right)-\mathcal{A}_{L}^{\top} e & =0, \\
\mathcal{A}_{v}^{\top} e & =v_{s},
\end{aligned}
$$

for the unknowns $e(t), i_{L}(t)$, and $i_{v}(t)$, where $e(t)$ denotes the vector containing the potential in the circuit nodes with respect to the mass node. Equation (4.1) is the 
Kirchhoff current law for the complete circuit, where the current-voltage relations for the resistors and capacitors have been included. Equation (4.2) describes the voltagecurrent characteristics of the inductors, and (4.3) determines the node potentials adjacent to the given voltage sources.

The semiconductor device is coupled to the electric network equations first by means of the semiconductor incidence matrix and the semiconductor current, and second via the boundary conditions for the electric potential in the semiconductor device. At terminal $k$, we have

$$
V(t)=e_{j}(t)+V_{\mathrm{bi}} \text { on } \Gamma_{k},
$$

if the terminal $k$ of the device is connected to the circuit node $j$, where $e_{j}$ denotes the potential at the circuit node $j$.

Equations (4.1)-(4.3) represent a system of differential-algebraic equations with a properly stated leading term $[20,28]$ if the matrices $C(v, t)=\left(\partial q_{C} / \partial v\right)(v, t)$ and $L(i, t)=\left(\partial \phi_{L} / \partial i\right)(i, t)$ are positive definite for all arguments $v, i$, and $t$. Under the assumptions that the matrices $C, L$, and $G=\partial g_{R} / \partial v$ are positive definite and that the circuit does neither contain loops of voltage sources only nor cutsets of current sources only, it is proved in $[36,38]$ that the (tractability) index of the DAE system is at most two. Furthermore, if the circuit does neither contain LI-cutsets nor CV-loops with at least one voltage source, the index is at most one.

4.2. Electro-thermal coupling. For the coupling between the electric and thermal network equations, we assume that only semiconductor devices and resistors are thermally relevant, as they are the most important elements exhibiting this behavior [31]. The thermal-to-electric coupling is accomplished by the temperaturedependent mobilities (2.1). Electric-to-thermal coupling occurs through the power dissipated by a resistor which is the product of the current through the resistor and the applied voltage. We assume an (at most) quadratic dependence of the resistance on the corresponding temperature $T_{R}$,

$$
R=1+\alpha_{1} T_{R}+\alpha_{2} T_{R}^{2}
$$

where $\alpha_{1}$ and $\alpha_{2}$ are some nonnegative parameters [7, Sec. 5.3]. The temperature values $T_{R}$ are collected in the vector $\boldsymbol{T}_{R}$ and can be determined from the thermal network by employing the matrices $\mathcal{K}=\left(k_{\ell j}\right) \in \mathbb{R}^{m_{d} \times n_{R}}$ and $\widehat{\mathcal{K}}=\left(\widehat{k}_{\ell j}\right) \in \mathbb{R}^{m_{n} \times n_{R}}$, defined by

$$
\begin{aligned}
& k_{\ell j}= \begin{cases}1 & \text { if the resistor } j \text { corresponds to the thermal branch } \ell \\
0 & \text { else, }\end{cases} \\
& \hat{k}_{\ell j}= \begin{cases}1 & \text { if the resistor } j \text { corresponds to the thermal node } \ell \\
0 & \text { else, }\end{cases}
\end{aligned}
$$

where $n_{R}$ is the number of resistive branches in the electric network. Notice that the resistors are considered as electrically lumped elements and thus, the temperature values are lumped, too. As an alternative, a resistor can be modeled by a thermally distributed element. In this case, we determine the lumped value $\widetilde{\boldsymbol{T}}_{j}^{d}$ from the distributed values $\boldsymbol{T}_{j}^{d}$ by simply taking the mean value. Then it holds

$$
\boldsymbol{T}_{R}=\widehat{\mathcal{K}}^{\top} \widehat{\boldsymbol{T}}+\mathcal{K}^{\top} \widetilde{\boldsymbol{T}}^{d}
$$


Now, the electric-to-thermal coupling is realized by the source terms $P=\left(P_{j}\right)$ and $\widehat{\boldsymbol{P}}$ occurring in the (lumped) heat flow equations (3.1) and (3.2). These terms represent the heat production caused by the dissipated power, which is given as the product of the current and the applied voltage, see $[3,7]$. With $i_{R}$ containing the current through all resistors, the power dissipated by all resistors in the electric network is given by

$$
\mathcal{P}_{R}(e)=\operatorname{diag}\left(i_{R}\right) \mathcal{A}_{R}^{\top} e \in \mathbb{R}^{n_{R}}
$$

where $\mathcal{A}_{R}$ denotes the resistor incidence matrix of the electric network and $e$ is the vector containing the node potentials. Observe that the entries of $\mathcal{P}_{R}$ are all lumped quantities. Thus, if the $j$-th resistor is thermally modeled as a distributed element, the lumped quantity has to be extended to a distributed one. The simplest choice to do this is to assume a constant local power dissipation within the resistor:

$$
\widetilde{\mathcal{P}}_{R, j}(x, t)=L_{R, j}^{-1} \mathcal{P}_{R, j} \quad \text { for } x \in\left(0, L_{R, j}\right),
$$

with the length $L_{R, j}$ of the distributed resistor. This corresponds to the assumption of a constant electric field inside the resistor. A more detailed discussion about the proper choice of the local power distribution can be found in [7], where local thermal effects and passivity of the elements are also taken into account.

With the matrices $\mathcal{K}$ and $\widehat{\mathcal{K}}$ defined in (4.5) and (4.6), we can express the electrothermal source terms occurring in (3.1) and (3.2) as

$$
\widehat{\boldsymbol{P}}=\widehat{\mathcal{K}} \mathcal{P}_{R}(e) \quad \text { and } \quad \boldsymbol{P}=\left(P_{1}, \ldots, P_{m_{d}}\right)=\mathcal{K} \widetilde{\mathcal{P}}_{R}(e)
$$

A summary of the coupled thermal device-circuit model can be found in the following section.

5. Scaling and the complete model. We scale the device model and summarize the complete thermo-electric network-device model.

Let $C_{m}$ be the maximal value of the doping profile, $L$ the device diameter, $\mu_{0}=$ $\max \left\{\mu_{n, 0}, \mu_{p, 0}\right\}$ the maximum of the low-field mobilities and $T_{0}=300 \mathrm{~K}$. Furthermore, let $U_{T}=k_{B} T_{0} / q$ be the thermal voltage and $t_{0}=L^{2} /\left(\mu_{0} U_{T}\right)$ be a typical time scale. We employ the following scaling:

$$
\begin{array}{llll}
x \rightarrow L x, & t \rightarrow t_{0} t, & \tau_{0} \rightarrow t_{0} \tau_{0}, & \tau_{n / p} \rightarrow t_{0} \tau_{n / p} \\
n \rightarrow C_{m} n, & p \rightarrow C_{m} p, & n_{i} \rightarrow C_{m} n_{i}, & C \rightarrow C_{m} C \\
T_{n} \rightarrow T_{0} T_{n}, & T_{L} \rightarrow T_{0} T_{L}, & \mu_{n, 0} \rightarrow \mu_{0} \mu_{n, 0}, & \mu_{p, 0} \rightarrow \mu_{0} \mu_{p, 0} \\
V \rightarrow U_{T} V, & V_{\text {app }} \rightarrow U_{T} V_{\mathrm{app}}, & V_{\mathrm{bi}} \rightarrow U_{T} V_{\mathrm{bi}}, & E_{c / v} \rightarrow k_{B} T_{0} E_{c / v}
\end{array}
$$

The Auger recombination parameters $C_{n}, C_{p}$ and the parameters in the heat flow equation are scaled as follows:

$$
\begin{array}{ll}
C_{n} \rightarrow\left(C_{m}^{2} t_{0}\right)^{-1} C_{n}, & C_{p} \rightarrow\left(C_{m}^{2} t_{0}\right)^{-1} C_{p}, \\
\kappa_{L} \rightarrow L^{2} k_{B} C_{m} t_{0}^{-1} \kappa_{L}, & \rho_{L} c_{L} \rightarrow k_{B} C_{m} \rho_{L} c_{L}, \quad S_{L} \rightarrow k_{B} C_{m} t_{0}^{-1} S_{L} .
\end{array}
$$

The time variables occurring in the electric and thermal network equations are scaled by $t_{0}$ as well. We remark that the semiconductor current and the heat flux have to be unscaled before being coupled to the network equations. For notational convenience we will not rename the scaled quantities in the following. 
It is convenient to formulate the energy-transport equations as in [15] by employing the variables $g_{n}=\widetilde{\mu}_{n} n, g_{w, n}=\frac{3}{2} \widetilde{\mu}_{n} n T_{n}, g_{p}=\widetilde{\mu}_{p} p$ and $g_{w, p}=\frac{3}{2} \widetilde{\mu}_{p} p T_{p}$, where the mobilities are given by

$$
\widetilde{\mu}_{n}=\mu_{n, 0} T_{L}^{1-\alpha_{n}}, \quad \widetilde{\mu}_{p}=\mu_{p, 0} T_{L}^{1-\alpha_{p}},
$$

and the numbers $\alpha_{n}$ and $\alpha_{p}$ are introduced in (2.1). In these variables, the energytransport system is of drift-diffusion type which allows us to discretize the equations similarly as the semiconductor drift-diffusion equations (see below).

The model equations and the corresponding coupling conditions are summarized in Tables 5.1 and 5.2. The parameter $\lambda$ is the scaled Debye length defined by $\lambda^{2}=$ $\varepsilon_{s} U_{T} /\left(q C_{m} L^{2}\right)$.

Electric network:

$$
\begin{aligned}
t_{0}^{-1} \mathcal{A}_{C} \frac{d q_{C}}{d t}\left(\mathcal{A}_{C}^{\top} e, t\right)+\mathcal{A}_{R} g_{R}\left(\mathcal{A}_{R}^{\top} e, \boldsymbol{T}_{R}\right)+\mathcal{A}_{L} i_{L}+\mathcal{A}_{v} i_{v}+\mathcal{A}_{S} j_{S} & =-\mathcal{A}_{i} i_{s}, \\
t_{0}^{-1} \frac{d \phi_{L}}{d t}\left(i_{L}, t\right)-\mathcal{A}_{L}^{\top} e & =0, \\
\mathcal{A}_{v}^{\top} e & =v_{s} .
\end{aligned}
$$

Thermal network:

$$
\begin{aligned}
t_{0}^{-1} M_{i} \partial_{t} T_{i}^{d} & =\partial_{x}\left(\kappa_{i} \partial_{x} T_{i}^{d}\right)-S_{i}\left(T_{i}^{d}-T_{\mathrm{env}}\right)+P_{i}, \quad i=1, \ldots, m_{d}, \\
t_{0}^{-1} \widehat{\boldsymbol{M}} \frac{d \widehat{\boldsymbol{T}}}{d t} & =\widehat{\boldsymbol{F}}^{S}+\widehat{\boldsymbol{F}}^{d}-\widehat{\boldsymbol{S}}\left(\widehat{\boldsymbol{T}}-T_{\mathrm{env}} \boldsymbol{I}\right)+\widehat{\boldsymbol{P}}, \\
\widehat{\boldsymbol{F}}^{d} & =\mathcal{A}_{d}^{\text {th }}\left(\begin{array}{c}
\Lambda(0) \partial_{x} \boldsymbol{T}^{d}(0, t) \\
-\Lambda\left(L_{\mathrm{th}}\right) \partial_{x} \boldsymbol{T}^{d}\left(L_{\mathrm{th}}, t\right)
\end{array}\right), \quad\left(\begin{array}{c}
\boldsymbol{T}_{0}^{d} \\
\boldsymbol{T}_{1}^{d}
\end{array}\right)=\left(\mathcal{A}_{d}^{\mathrm{th}}\right)^{\top} \widehat{\boldsymbol{T}} .
\end{aligned}
$$

Semiconductor equations:

$$
\begin{gathered}
\lambda^{2} \Delta V=\widetilde{\mu}_{n}^{-1} g_{n}-\widetilde{\mu}_{p}^{-1} g_{p}-C(x), \\
\widetilde{\mu}_{n}^{-1} \partial_{t} g_{n}-\operatorname{div} J_{n}=-R(n, p), \\
\widetilde{\mu}_{n}^{-1} \partial_{t} g_{w, n}-\operatorname{div} S_{n}=-\nabla V \cdot J_{n}+W_{n}\left(g_{n}, g_{w, n}\right)-\frac{3}{2} T_{n} R(n, p), \\
\widetilde{\mu}_{p}^{-1} \partial_{t} g_{p}+\operatorname{div} J_{p}=-R(n, p), \\
\widetilde{\mu}_{p}^{-1} \partial_{t} g_{w, p}+\operatorname{div} S_{p}=-\nabla V \cdot J_{p}+W_{p}\left(g_{p}, g_{w, p}\right)-\frac{3}{2} T_{p} R(n, p), \\
J_{n}=\nabla g_{n}-\frac{g_{n}}{T_{n}} \nabla V, \quad S_{n}=\nabla g_{w, n}-\frac{g_{w, n}}{T_{n}} \nabla V, \\
J_{p}=-\nabla g_{p}-\frac{g_{p}}{T_{p}} \nabla V, \quad S_{p}=-g_{w, p}-\frac{g_{w, p}}{T_{p}} \nabla V, \\
\rho_{L} c_{L} \partial_{t} T_{L}-\operatorname{div}\left(\kappa_{L} \nabla T_{L}\right)=-\left(W_{n}+W_{p}\right)+R\left(E_{g}+\frac{3}{2}\left(T_{n}+T_{p}\right)\right)-S_{L}\left(T_{L}-T_{\mathrm{env}}\right) .
\end{gathered}
$$

TABLE 5.1

Scaled coupled thermo-electric network-device model equations.

6. Numerical examples. In this section, the coupled thermo-electric model is numerically discretized and two numerical examples are presented. In view of the complexity of the coupled system, the semiconductor device is described by a 
Electric-thermal network interface:

$$
\begin{aligned}
\boldsymbol{T}_{R} & =\widehat{\mathcal{K}}^{\top} \widehat{\boldsymbol{T}}+\mathcal{K}^{\top} \widetilde{\boldsymbol{T}}^{d}, \quad \widetilde{\boldsymbol{T}}^{d}=\operatorname{mean}\left(\boldsymbol{T}^{d}\right), \\
\widehat{\boldsymbol{P}} & =\widehat{\mathcal{K}}\left(\operatorname{diag}\left(i_{R}\right) \mathcal{A}_{R}^{\top} e\right), \quad P_{j}=\left(\mathcal{K} \widetilde{\mathcal{P}}_{R}(e)\right)_{j}, \\
\left(\widetilde{\mathcal{P}}_{R}(e)\right)_{j} & =L_{R}^{-1}\left(\operatorname{diag}\left(i_{R}\right) \mathcal{A}_{R}^{\top} e\right)_{j}, \quad j=1, \ldots, m_{d} .
\end{aligned}
$$

Circuit-device interface:

$$
\begin{aligned}
j_{S, k} & =L\left(q \mu_{0} U_{T} C_{m}\right)^{-1} \int_{\Gamma_{k}}\left(J_{n}+J_{p}+J_{d}\right) \cdot \nu d \sigma \quad \text { with } J_{d}=-\lambda^{2} \nabla \partial_{t} V, \\
V(\cdot, t) & =U_{T}^{-1} e_{i}(t)+V_{\mathrm{bi}}(\cdot, t) \quad \text { on } \Gamma_{k}, k=1, \ldots, n_{S} .
\end{aligned}
$$

Thermal network-device interface:

$$
\begin{aligned}
\widehat{\boldsymbol{F}}^{\mathrm{S}} & =\mathcal{A}_{S}^{\mathrm{th}}\left(F_{1}^{S}(t), \ldots, F_{n_{S}}^{S}(t)\right), \quad \text { with } F_{k}^{S}=\int_{\Gamma_{k}} J_{\mathrm{th}}^{S} \cdot \nu d \sigma, \\
J_{\mathrm{th}}^{S} & =L\left(q \mu_{0} U_{T}^{2} C_{m}\right)^{-1}\left(-\kappa_{L} \nabla T_{L}-\left(E_{c} J_{n}+E_{v} J_{p}\right)-S_{n}+S_{p}\right), \\
\boldsymbol{T}_{a} & =\mathcal{A}_{S}^{\mathrm{th}^{\top}} \widehat{\boldsymbol{T}} T_{0}^{-1} .
\end{aligned}
$$

Coupling conditions for the thermo-electric network-device model.

spatially one-dimensional model. The electron transport is modeled by the energytransport equations, the hole transport by the drift-diffusion equations. Thus, the hole temperature is assumed to be constant.

6.1. Numerical scheme. The electric network model consists of differentialalgebraic equations. The thermal network equations are of partial differential (algebraic) type, depending on whether artificial zero-dimensional units occur or not. The device is modeled by a system of nonlinear elliptic and parabolic differential equations. Thus the complete thermo-electric model is a system of nonlinear partial differential-algebraic equations.

The entire system is first discretized in time by the 2-stage backward difference formula as in $[11,12]$. This method takes into account the differential-algebraic character of the problem. The heat equations describing the thermal lines and the lattice temperature are discretized by standard finite elements. The energy-transport and drift-diffusion equations are approximated by exponentially fitted hybrid-mixed finite elements. We employ the finite elements of Marini and Pietra [29] since this guarantees particle current continuity and positive particle densities for the discrete problem provided that a certain step size restriction holds. We refer to [11] for details of the finite-element discretization.

The nonlinear discrete system is solved iteratively as follows. In the outer loop, solve (1) the semiconductor transport equations including the heat equation, (2) the electric network equations, (3) the thermal network equations, and update (4) the boundary conditions for the lattice temperature and the temperature-dependent electrical parameters until the difference of two successive iterations is smaller than a prescribed tolerance value. The nonisothermal semiconductor transport equations in (1) are solved in an inner loop using a variant of the Gummel method (see [11]). More 


\begin{tabular}{|l|l|l|}
\hline Parameter & Physical meaning & Numerical value \\
\hline$L_{y}$ & extension of the device in $y$-direction & $6 \cdot 10^{-7} \mathrm{~m}$ \\
$L_{z}$ & extension of the device in $z$-direction & $10^{-6} \mathrm{~m}$ \\
$q$ & elementary charge & $1.6 \cdot 10^{-19} \mathrm{As}$ \\
$\varepsilon_{s}$ & permittivity constant & $10^{-12} \mathrm{As} / \mathrm{Vcm}$ \\
$U_{T}$ & thermal voltage at $T_{L}=300 K$ & $0.026 \mathrm{~V}$ \\
$\mu_{n, 0} / \mu_{p, 0}$ & low-field carrier mobilities $[27]$ & $1500 / 450 \mathrm{~cm}^{2} / \mathrm{Vs}$ \\
$\tau_{n} / \tau_{p}$ & carrier lifetimes $[27]$ & $10^{-6} / 10^{-5} \mathrm{~S}$ \\
$C_{n} / C_{p}$ & Auger parameters $[27]$ & $1.1 \cdot 10^{-42} / 0.3 \cdot 10^{-42} \mathrm{~m}^{6} / \mathrm{s}$ \\
$n_{i}$ & intrinsic density [27] & $10^{16} \mathrm{~m}^{-3}$ \\
$\tau_{n, 0}$ & energy relaxation time $[15]$ & $4 \cdot 10^{-13} \mathrm{~S}$ \\
$\kappa_{L}$ & thermal conductivity $[27]$ & $130 \mathrm{~W} / \mathrm{mK}$ \\
$c_{L}$ & heat capacity [27] & $700 \mathrm{~J} / \mathrm{kgK}$ \\
$\rho_{L}$ & material density [27] & $2329 \mathrm{~kg} / \mathrm{m}^{3}$ \\
$\alpha_{n} / \alpha_{p}$ & mobility power-law parameters & 2 \\
$R_{\mathrm{th}}$ & thermal contact resistance $[18]$ & $0 \ldots 10^{-7} \mathrm{~s} \mathrm{~m} / \mathrm{W}$ \\
$S_{L}$ & radiation parameter & $0 \ldots 4 \cdot 10^{15} \mathrm{~W} / \mathrm{m}^{3} \mathrm{~K}$ \\
\hline
\end{tabular}

Physical parameters for a silicon diode.

precisely, (1) consists of the following steps: (1a) solve the transport equations for the electrons and holes and the Poisson equation using the Gummel method, (1b) solve the energy equation, (1c) update the electron temperature, (1d) solve the Poisson equation and the heat equation for the lattice temperature, and (1e) update the carrier mobilities.

6.2. Lattice temperature in a ballistic diode. As first numerical example we consider a one-dimensional $n^{+} n n^{+}$ballistic diode. The diode can be considered as a simple model of the channel of a MOS transistor. The diode with domain $\Omega=(0, L)$, where $L=600 \mathrm{~nm}$, consists of a $400 \mathrm{~nm} n$-channel with minimal doping of $2 \cdot 10^{21} \mathrm{~m}^{-3}$ and two adjacent $100 \mathrm{~nm} n^{+}$-regions with a maximal doping of $5 \cdot 10^{23} \mathrm{~m}^{-3}$. The doping profile is smoothed by use of the tanh function. The device is modeled by the unipolar energy-transport equations coupled to the Poisson equation and the heat equation for the lattice temperature. In the unipolar case, the source term in the heat equation simplifies to $H=-W_{n}-S_{L}\left(T_{L}-T_{n}\right)$ since there are no recombination effects. The physical parameters used in the simulation are collected in Table 6.1.

At the boundary nodes $x=0$ and $x=L$, we assume Robin conditions for the electron density and homogeneous Neumann conditions for the electron temperature:

$$
J_{n} \cdot \nu=\theta_{n}\left(g_{n, a}-g_{n}\right), \quad \nabla T_{n} \cdot \nu=0 \quad \text { on } \Gamma_{C}, t>0,
$$

where $g_{n, a}=\mu_{n} n_{a}, n_{a}$ is defined in (2.7), and we have chosen $\theta_{n}=2500$ [11]. We compare two different boundary conditions for the lattice temperature:

$$
T_{L}=T_{0}=1 \quad \text { or } \quad-\kappa_{L} \nabla T_{L} \cdot \nu=R_{\mathrm{th}}^{-1}\left(T_{L}-T_{0}\right) \quad \text { on } \Gamma_{C}, t>0
$$

where $R_{\mathrm{th}}=10^{-8} \mathrm{sm}^{2} / \mathrm{W}$. Finally, at $t=0$ we assume that the device is in thermal equilibrium,

$$
g_{n}=\mu_{n} n_{\mathrm{eq}}, \quad V=V_{\mathrm{eq}}, \quad T_{n}=1, \quad T_{L}=1 \quad \text { in } \Omega,
$$


where $\left(n_{\mathrm{eq}}, V_{\mathrm{eq}}\right)$ are the thermal equilibrium values of the semiconductor (see, e.g., $[22,30])$. We perform the simulations on a spatial grid of 101 nodes with a time step of $2 \cdot 10^{-13} \mathrm{~s}$.

Figure 6.1 illustrates the transient behavior of the lattice temperature in the ballistic diode biased with $1.5 \mathrm{~V}$ and without radiation. We observe a temperature increase of less than $1 \mathrm{~K}$ when we employ Dirichlet boundary conditions (Figure 6.1 left) which coincides with the results presented in [32]. The Dirichlet condition implies an infinite heat flux across the boundary which is physically questionable. With the Robin conditions for the lattice temperature, thus taking into account a nonvanishing thermal resistance of the contacts, the temperature increase is more pronounced (Figure 6.1 right). Inside the device, the lattice temperature variations are small which comes from the fact that the heat conductivity is rather high. In the following, the simulations are performed using the Robin boundary conditions for $T_{L}$.
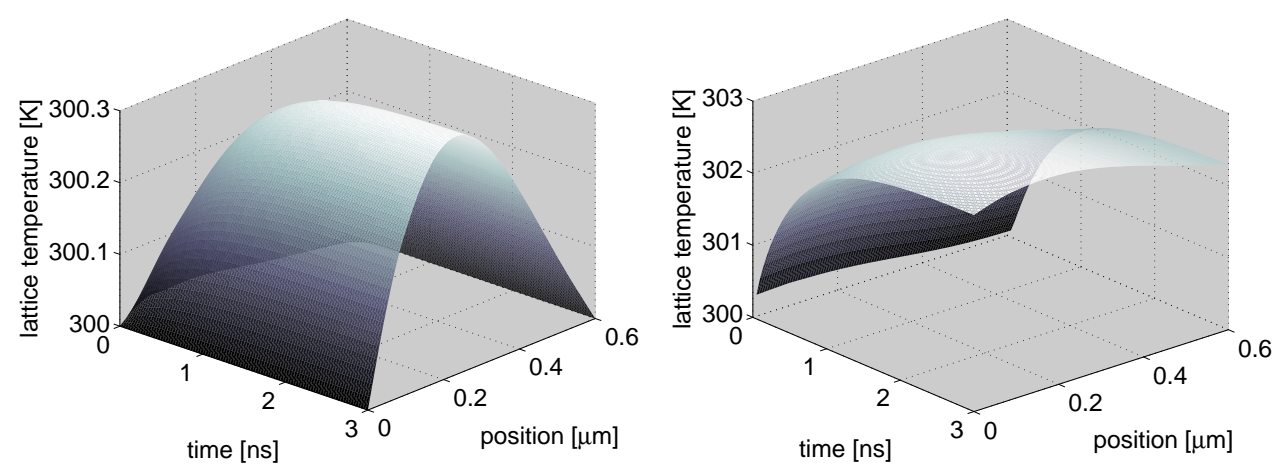

FIG. 6.1. Lattice temperature in a ballistic diode with $400 \mathrm{~nm}$ channel biased with $1.5 \mathrm{~V}$. Left: Dirichlet boundary conditions, right: Robin boundary conditions.

It is well known in industrial applications that thermal effects in semiconductors are becoming stronger in smaller devices. For comparison, we simulate a diode with length $L=250 \mathrm{~nm}$, consisting of a $50 \mathrm{~nm}$ channel and two $100 \mathrm{~nm} n^{+}$-regions on each side. The doping concentration is as in the previous example. In the literature, this device was numerically simulated using an energy-transport model [4] and a hydrodynamic model [23], in both cases, however, with constant lattice temperature only. We apply the voltage $V_{\text {app }}=1 \mathrm{~V}$. For the radiation parameter we choose $S_{L}=$ $4 \cdot 10^{15} \mathrm{~W} / \mathrm{m}^{3} \mathrm{~K}$. With an extension of $250 \mathrm{~nm}$ in the $y$ - and $z$-directions, this value corresponds to a transmission value of approximately $6 \cdot 10^{-4} \mathrm{~W} / \mathrm{K}$ for the entire device which is much larger than realistic physical values (cf. [7]). The number of grid points is as above but we have chosen a time step of $10^{-14} \mathrm{~s}$ in order to resolve the transient response of the device shortly after the abrupt increase of the bias and to preserve the positivity of the particle densities in the device model.

The lattice temperature $T_{L}$ of the device is shown in Figure 6.2 (left). We observe an increase of $T_{L}$ of about $5 \mathrm{~K}$ assuming a very high radiation to the environment. The lattice temperature computed from the model without radiation is presented in Figure 6.2 (right). The lattice temperature maximum is about $325 \mathrm{~K}$ compared to $302 \mathrm{~K}$ in the $400 \mathrm{~nm}$ channel device. This clearly shows the importance of self-heating in small devices.

In order to clarify the impact of the lattice heating on the electrical behavior of the device, we compare the current-voltage characteristics computed from the energy- 

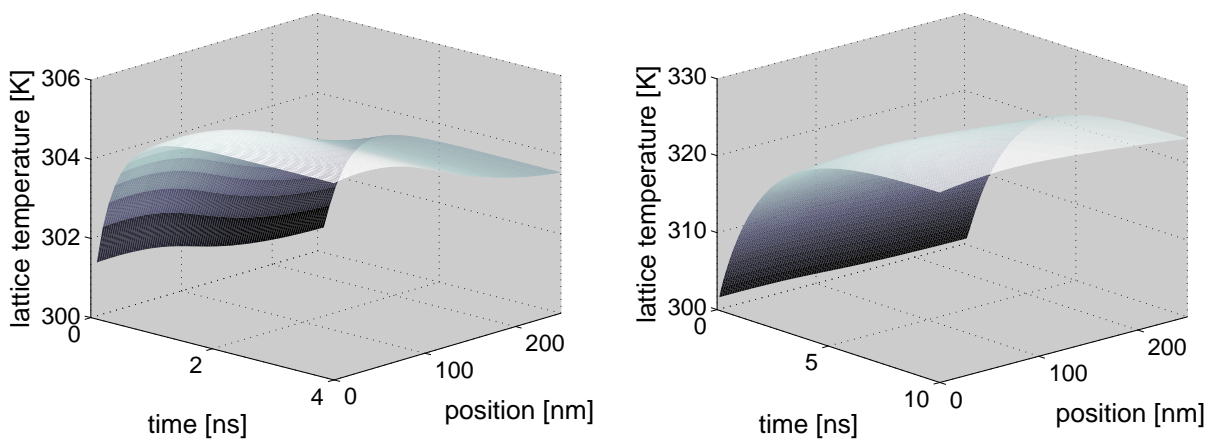

FIG. 6.2. Lattice temperature in a ballistic diode with $50 \mathrm{~nm}$ channel biased with $1 \mathrm{~V}$ including radiation (left) and without radiation (right).
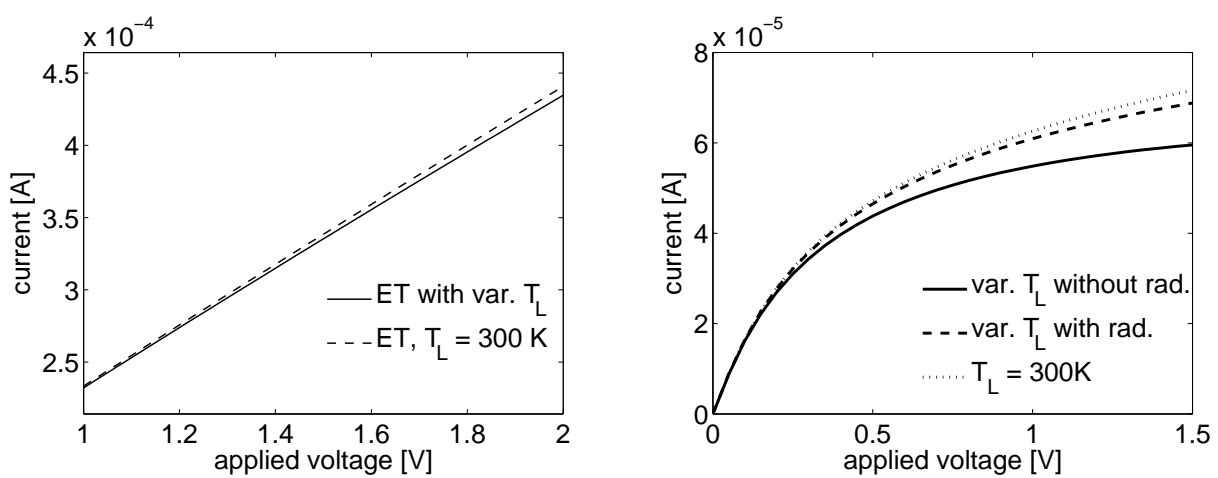

FIG. 6.3. Current-voltage characteristics for ballistic diodes computed from the drift-diffusion model and the energy-transport (ET) model with constant and variable lattice temperature for the $400 \mathrm{~nm}$ channel (left) and $50 \mathrm{~nm}$ channel (right) device.

transport model with constant lattice temperature to those computed from the model with variable lattice temperature, see Figure 6.3. For the $400 \mathrm{~nm}$ channel device, for which we have neglected radiation, we observe that the influence of the model on the current-voltage characteristics is rather small and becomes significant for very high applied bias only (Figure 6.3 left). This shows that for larger devices the influence of lattice heating seems to be less important.

On the other hand, the influence of the lattice temperature on the current-voltage curve becomes stronger for smaller devices, even for rather small applied voltages. In Figure 6.3 (right) we also see the effect of radiation on the device performance. We notice that we model the thermal radiation by the expression $S_{L}\left(T_{L}-T_{\text {env }}\right)$ with constant environmental temperature. In applications, this is not always satisfied, as the environmental temperature might increase due to continuous radiation from the electric circuit elements. An increase of $T_{\text {env }}$, in turn, decreases the thermal radiation which would again increase lattice heating and influences further the device performance. Moreover, the considered radiation parameter is chosen very high and might be unrealistic.

6.3. Frequency multiplier. The second numerical example is concerned with the transient simulation of a frequency multiplier which is used in, for instance, radio 


\begin{tabular}{|l|l|l|}
\hline Parameter & Physical meaning & Numerical value \\
\hline$R_{1} / R_{2}$ & resistances & $100 / 1000 \Omega$ \\
$C_{1}$ & capacitance & $(3.2 \cdot 2 \pi)^{-1} \cdot 10^{-10} \mathrm{~F}$ \\
$L_{1}$ & inductance & $(3.2 \cdot 2 \pi)^{-1} \cdot 10^{-6} \mathrm{H}$ \\
$\kappa_{R}$ & heat conductivity of resistor & $237 \mathrm{~W} / \mathrm{mK}$ \\
$c_{R}$ & heat capacity of resistor & $900 \mathrm{~J} / \mathrm{kgK}$ \\
$\rho_{R}$ & material density of resistor & $2700 \mathrm{~kg} / \mathrm{m}^{3}$ \\
$L_{R}$ & length of resistor & $10^{-6} \mathrm{~m}$ \\
$A_{R}$ & cross section of resistor & $10^{-7} \mathrm{~m}$ \\
$\alpha_{1}$ & first thermal coefficient & $1 /(273 \mathrm{~K})$ \\
$\alpha_{2}$ & second thermal coefficient & $1 /(273 \mathrm{~K})^{2}$ \\
\hline
\end{tabular}

Electrical and thermal parameters of the frequency multiplier. The thermal coefficients $\alpha_{1}$ and $\alpha_{2}$ occur in the definition (4.4) of the resistance.

receivers and digital electronics (see Figure 6.4). The multiplier is modeled by the complete thermo-electric network-device system. The circuit consists of a voltage source generating an oscillatory signal with the eigenfrequency of the first oscillator. The signal is modified by the $p n$ diode. As we choose the capacity $C_{2}=C_{1} / 2$ and the inductivity $L_{2}=L_{1} / 2$, the second oscillator resonates with the double frequency and other frequencies are damped out. We employ the voltage signal $v(t)=3 \mathrm{~V} \sin (3.2$. $2 \pi \cdot 10^{9} \mathrm{~Hz} t$ ) such that the first oscillator resonates at the frequency of $3.2 \mathrm{GHz}$.

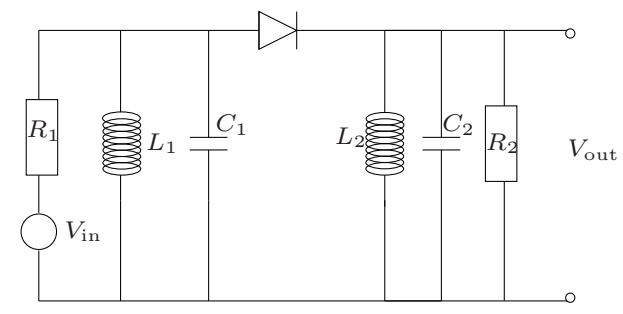

FIG. 6.4. Frequency multiplier.

As mentioned at the beginning of section 4.2 , only the resistors and the diode are treated as thermally relevant circuit elements. The resistors are modeled as thermal lines. The diode is modeled by the energy-transport model for the electrons and the drift-diffusion model for the holes described in section 2.1. Thus, the only thermally lumped elements are the artificial nodes modeling the contacts between the resistors and the device. We notice that the temperature at the contacts coincides with the temperature at the boundary of the thermal lines. For the device, however, the temperature at the contacts is described by Robin boundary conditions. The electrical and thermal parameters of the circuit elements are collected in Table 6.2.

The $p n$ diode is assumed to be homogeneous in the $y$ - and $z$-directions such that a one-dimensional approach is suitable. The diode consists of an $n$-doped region with $50 \mathrm{~nm}$ length and maximal doping concentration of $C_{0}=3 \cdot 10^{23} \mathrm{~m}^{-3}$ and a $p$-region of the same length and minimal doping density of $-C_{0}$. We take the thermal radiation parameter $S_{L}=4 \cdot 10^{15} \mathrm{~W} / \mathrm{m}^{3} \mathrm{~K}$ and the thermal contact resistance $R_{\mathrm{th}}=$ $10^{-8} \mathrm{~s} \mathrm{~m}^{2} / \mathrm{W}$. The remaining parameters can be found in Table 6.1.

Figure 6.5 illustrates the current through the diode and the output signal of the 
circuit. The results including thermal effects (computed from the energy-transport model with lattice heating) are compared with those without thermal effects (computed from the drift-diffusion equations with constant lattice temperature). Due to lattice heating, the current through the diode becomes smaller, but the effect is not large in the output signal since the output voltage is rather small.
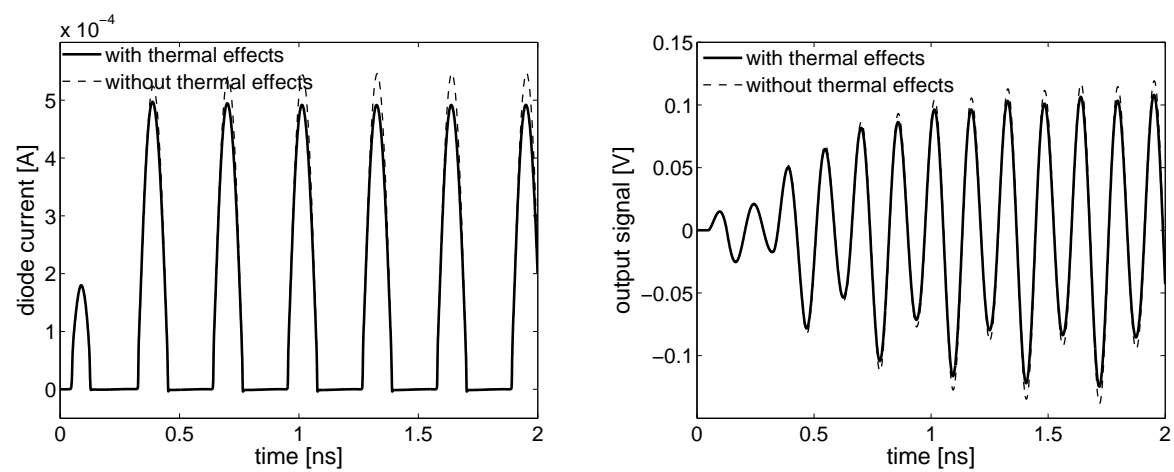

FIG. 6.5. Diode current (left) and output signal (right) of the frequency multiplier.

The transient behavior of the distributed temperatures are depicted in Figures 6.6 and 6.7. In Figure 6.6, we observe an oscillatory behavior of the lattice temperature caused by the oscillatory input signal. The maximum of the lattice temperature is increasing before stabilizing. The resistors, on the other hand, are much less heating up than the diode, as shown in Figure 6.7. After 2 nanoseconds, the increase of the lattice temperature is less than $0.1 \mathrm{~K}$. Hence, its influence on the resistivity is small and the current in the first oscillator remains almost unchanged. It is interesting to observe that the temperatures in the resistors are maximal at those contacts which are connected to the diode, caused by the heat exchange with the bipolar diode.

7. Conclusions. In this paper, we have presented a fully coupled thermo-electric network-device model consisting of three subsystems: the electric network, the thermal network, and the semiconductor device. The first subsystem was modeled by the electric network equations from modified nodal analysis, the second system describes the temperatures of the lumped and distributed network elements using heat equation models, and the third subsystem was modeled by the bipolar energy-transport equations including a heat equation for the lattice temperature. Coupling conditions between these subsystems were developed.

The heat equation for the lattice temperature was derived from thermodynamic principles following $[2,6]$ which guarantees conservation of the total energy. The source term in the heat equation contains energy relaxation, recombination heat, and radiation effects. In order to couple the heat flux of the device to the thermal network, an expression for the semiconductor heat flux, based on a quasi-stationarity assumption for the energy balance equation, was derived and employed to define the thermal flux in the heat equation for the temperatures of the thermal nodes.

The energy-transport model for electrons with lattice heating was employed to simulate a one-dimensional ballistic diode. The numerial results confirm the physical observation that thermal effects play an important role in small semiconductor devices with channel lengths of much less than $1 \mu \mathrm{m}$. A second example was concerned with the simulation of a frequency multiplier containing a $p n$-junction diode. Lattice 


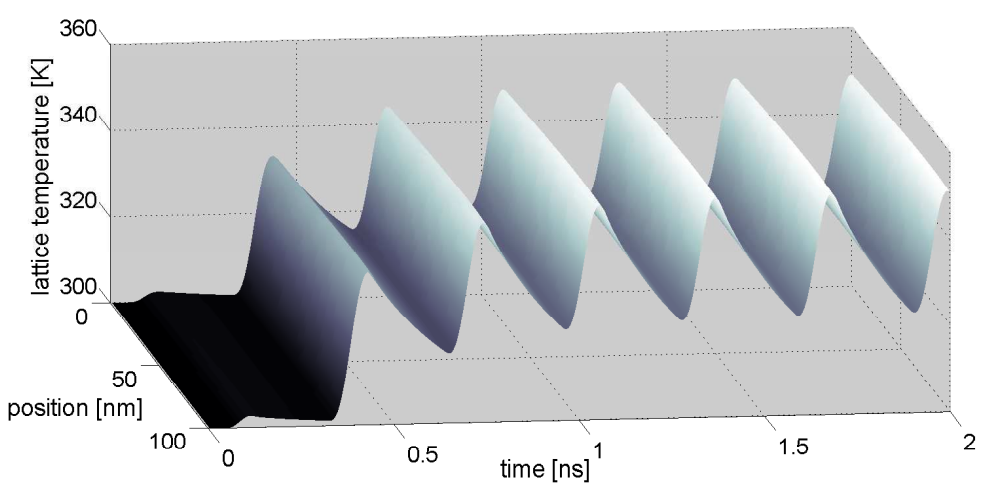

FIG. 6.6. Lattice temperature of the diode in the frequency multiplier.
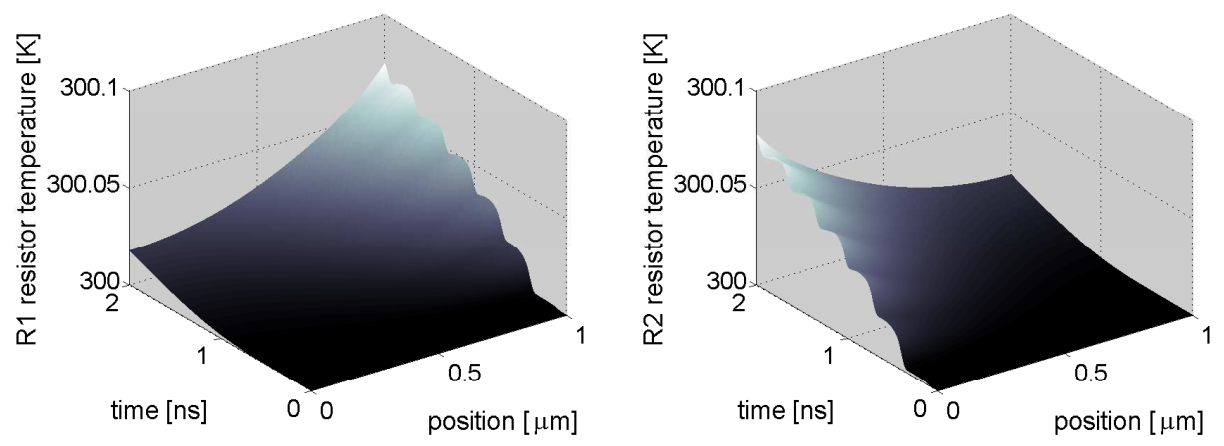

FIG. 6.7. Temperature of the resistors $R 1$ (left) and R2 (right) in the frequency multiplier.

heating causes a decrease of the diode current and a slight decrease of the output signal. The diode heats up significantly, whereas the thermal effects seem to be of minor importance in the resistors.

Due to the partial differential-algebraic structure, it seems to be possible to incorporate our model in existing electric circuit solvers (for instance, in TITAN). The numerical results seem to indicate, at least for the presented circuit, that the index of the system does not increase even for the fully coupled model. Future work may be concerned with circuit-device systems containing several devices and the simulation of MOS transistors in several space dimensions [21] and silicon-on-insulator (SOI) structures [9].

\section{REFERENCES}

[1] M. AdLER, Accurate calculations of the forward drop and power dissipation in thyristors, IEEE Trans. Electr. Dev., 25 (1978), pp. 16-22.

[2] G. Albinus, H. Gajewski, and R. Hünlich, Thermodynamic design of energy models of semiconductor devices, Nonlinearity, 15 (2002), pp. 367-383.

[3] G. Alì And M. CARINI, Energy-transport models for semiconductor devices and their coupling with electric networks, to appear in Proceedings of WSCP 2006, 2008.

[4] A. Anile, A. Marrocco, V. Romano, and J. Sellier, $2 D$ numerical simulation of the MEP energy-transport model with a finite elements scheme, J. Comput. Electr., 4 (2005), pp. 231-259. 
[5] A. Anile, V. Romano, and G. Russo, Extendend hydrodynamic model of carrier transport in semiconductors, SIAM J. Appl. Math., 61 (2000), pp. 74-101.

[6] U. Bandelow, H. Gajewski, and R. Hünlich, Fabry-Perot lasers: thermodynamic-based modeling, in Optoelectronic Devices. Advanced Simulation and Analysis, J. Piprek (ed.), Springer, Berlin, pp. 63-85, 2005.

[7] A. Bartel, Partial Differential-Algebraic Models in Chip Design - Thermal and Semiconductor Problems, Ph.D. Thesis, Universität Karlsruhe, Germany, 2003.

[8] A. BARTEl AND M. GÜNThER, Multirate co-simulation of first order thermal models in electric circuit design, in Scientific Computing in Electrical Engineering. Proceedings SCEE 2002, W. Schilders et al. (eds.), Springer, Berlin, pp. 23-28, 2002.

[9] A. Bartel and M. Günther, From SOI to abstract electric-thermal-1D multiscale modeling for first order thermal effects, Math. Comput. Modell. Dynam. Syst., 9 (2003), pp. 25-44.

[10] N. Ben Abdallah And P. Degond, On a hierarchy of macroscopic models for semiconductors, J. Math. Phys., 37 (1996), pp. 3308-3333.

[11] M. BRunk AND A. Jüngel, Numerical coupling of electric circuit equations and energytransport models for semiconductors, SIAM J. Sci. Comput., 30 (2008), pp. 873-894.

[12] M. BRUnK AND A. JÜNGEL, Simulation of thermal effects in optoelectronic devices using coupled energy-transport and circuit models, to appear in Math. Models Meth. Appl. Sci., 2009.

[13] D. Chen, E. Kan, U. Ravaioli, C. Shu, and R. Dutton, An improved energy transport model including nonparabolicity and non-Maxwellian distribution effects, IEEE Electr. Dev. Letters, 13 (1992), pp. 26-28.

[14] A. Chryssafis AND W. Love, A computer-aided analysis of one-dimensional thermal transients in n-p-n power transistors, Solid-State Electron., 22 (1979), pp. 249-256.

[15] P. Degond, A. JÜngel, And P. Pietra, Numerical discretization of energy-transport models for semiconductors with non-parabolic band structure, SIAM J. Sci. Comput., 22 (2000), pp. 986-1007.

[16] K. Einwich, P. Schwarz, P. Trappe, And H. ZoJer, Simulatorkopplung für den Entwurf komplexer Schaltkreise der Nachrichtentechnik, in 7. ITG-Fachtagung "Mikroelektronik für die Informationstechnik", Chemnitz, pp. 139-144, 1996.

[17] S. Gaur and D. NAVon, Two-dimensional carrier flow in a transistor structure under nonisothermal conditions, IEEE Trans. Electr. Dev., 23 (1976), pp. 50-57.

[18] T. Grasser, Mixed-Mode Device Simulation, Ph.D. Thesis, Vienna University of Technology, Austria, 1999

[19] T. Grasser, T.-W. Tang, H. Kosina, and S. Selberherr, A review of hydrodynamic and energy-transport models for semiconductor device simulation, Proc. IEEE, 91 (2003), pp. $251-274$.

[20] I. Hiqueras And R. März, Differential algebraic systems with properly stated leading terms, Comput. Math. Appl., 48 (2004), pp. 215-235.

[21] S. Holst, A. JÜngel, AND P. PIETRA, An adaptive mixed scheme for energy-transport simulations of field-effect transistors, SIAM J. Sci. Comput., 25 (2004), pp. 1698-1716.

[22] A. JüNGEL, Transport Equations for Semiconductors, Lecture Notes in Physics, Springer, to appear, 2009.

[23] A. JÜNGEL AND S. TANG, A relaxation scheme for the hydrodynamic equations for semiconductors, Appl. Numer. Math., 56 (2002), pp. 899-915.

[24] M. KNAIPP, Modellierung von Temperatureinflüssen in Halbleiterbauelementen, Ph.D. Thesis, Vienna University of Technology, Austria, 1998.

[25] R. Lamour, Index determination and calculation of consistent initial values for DAEs, Comput. Math. Appl., 50 (2005), pp. 1125-1140.

[26] J. Litsios, B. Schmithüsen, U. Krumbein, A. Schenk, E. Lyumkis, B. Polsky, and W. Fichtner, DESSIS 3.0 Manual, ISE Integrated Systems Engineering, Zürich, 1996.

[27] M. Levinshtein, Handbook Series on Semiconductor Parameters, World Scientific, London, 1996.

[28] R. März, Differential algebraic systems anew, Appl. Numer. Math., 42 (2002), pp. 315-335.

[29] L. D. Marini and P. Pietra, New mixed finite element schemes for current continuity equations, COMPEL, 9 (1990), pp. 257-268.

[30] P. Markowich, C. Ringhofer, and C. Schmeiser, Semiconductor Equations, Springer, Vienna, 1990.

[31] G. Massobrio and P. Antognetti, Semiconductor Device Modeling with SPICE, 2nd ed., McGraw-Hill, New York, 1993.

[32] E. Medina And M. PAGani, Multi-physics modeling and numerical simulation of electrothermal effects in semiconductor devices, Master Thesis, Politecnico di Milano, Italy, 2006.

[33] S. Selberherr, Analysis and Simulation of Semiconductor Devices, Springer, Vienna, 1984. 
[34] M. Selva Soto and C. Tischendorf, Numerical analysis of DAEs from coupled circuit and semiconductor simulation, Appl. Numer. Math., 53 (2005), pp. 471-488.

[35] D. Sharma and K. Ramanathan, Modeling thermal effects on MOS I-V characteristics, IEEE Electr. Dev. Lett., EDL-4 (1983), pp. 362-364.

[36] C. Tischendorf, Topological index calculation of differential-algebraic equations in circuit simulation, Surv. Math. Industr., 8 (1999), pp. 187-199.

[37] C. TischendorF, Modeling circuit systems coupled with distributed semiconductor equations, in Modeling, Simulation, and Optimization of Integrated Circuits, K. Antreich, R. Bulirsch, A. Gilg, and P. Rentrop (eds.), Internat. Ser. Numer. Math., 146 (2003), pp. 229-247.

[38] C. Tischendorf, Coupled systems of differential algebraic and partial differential equations in circuit and device simulations, Habilitation thesis, Humboldt-Universität zu Berlin, Germany, 2003.

[39] G. WACHUTKA, Rigorous thermodynamic treatment of heat generation and conduction in semiconductor device modeling, IEEE Trans. Comp. Aided Design, 9 (1990), pp. 1141-1149.

[40] G. WAChutKa, Consistent treatment of carrier emission and capture kinetices in electrothermal and energy transport models, Microelectr. J., 26 (1995), pp. 307-315.

[41] A. Yamnahakki, Second-order boundary conditions for the drift-diffusion equations for semiconductors, Math. Models Meth. Appl. Sci., 5 (1995), pp. 429-455. 